\title{
Focus on Stem Cells in Human Reproduction
}

\section{Human ovarian cancer stem cells}

\author{
Sharmila A Bapat \\ National Centre for Cell Science, NCCS Complex, Pune University Campus, Ganeshkhind, Pune 411 007, India \\ Correspondence should be addressed to S Bapat; Email: sabapat@nccs.res.in
}

\begin{abstract}
The isolation and identification of stem-like cells in solid tumors or cancer stem cells (CSCs) have been exciting developments of the last decade, although these rare populations had been earlier identified in leukemia. CSC biology necessitates a detailed delineation of normal stem cell functioning and maintenance of homeostasis within the organ. Ovarian CSC biology has unfortunately not benefited from a pre-established knowledge of stem cell lineage demarcation and functioning in the normal organ. In the absence of such information, some of the classical parameters such as long-term culture-initiating assays to isolate stem cell clones from tumors, screening and evaluation of other epithelial stem cell surface markers, dye efflux, and label retention have been applied toward the putative isolation of CSCs from ovarian tumors. The present review presents an outline of the various approaches developed so far and the various perspectives revealed that are now required to be dealt with toward better disease management.

Reproduction (2010) 140 33-41
\end{abstract}

\section{Introduction}

The last decade has witnessed a transition from a state of assumptions and hypothetical derivations relating to the involvement of stem cells in the onset and progression of cancer to the establishment of actual experimental proof of the isolation and identification of 'cancer stem cells' (CSCs) in several tumors (Lapidot et al. 1994). Notably, these include studies carried out in leukemia, breast, brain, ovary, prostate, pancreas, and others, a list that continues to expand rapidly in witness to the acceptance of the concept (Al-Haji et al. 2003, Singh et al. 2004, Bapat et al. 2005, Collins et al. 2005, Li et al. 2009). CSCs are considered especially significant on the background of drug resistance, tumor dormancy, minimal residual disease, and disease relapse. Their basic characteristics define very plausible mechanisms of quiescence that enable them to resist and evade therapy, and further, under optimal conditions, to draw upon their regenerative capabilities through self-renewal. This often leads to the emergence of a drug refractile and aggressive phase of disease. The understanding of such an involvement of CSCs has significant therapeutic implications and presents a major challenge to elucidate the intricate mechanisms of cell survival under adverse conditions. It thus becomes imperative to understand these cells from a developmental and evolutionary

This paper is one of four papers that form part of a special Focus Issue section on Stem Cells in Human Reproduction. The Guest Editor for this section was C E Gargett, Clayton, Australia. aspect that may be crucial in identifying the aberrant events leading to disease.

Lineage mapping and a clear correlation with surface markers that thus aids the identification of specific stem and progenitor subsets have been achieved to a great extent in the hematopoietic system, or in organs such as breast and brain. Using the same markers, CSCs were thereby isolated and studied in the corresponding tumors. Unfortunately, this approach cannot be directly applied in case of ovarian cancer where there is clearly a lack of information regarding the stem cell population in normal ovarian tissue. Over the last few years, several studies have focused on the isolation, identification, and characterization of stem cells from normal ovarian tissue as well as from tumor samples. Regenerative potential in the ovary has been associated with the ovarian surface epithelium (OSE) which was previously referred to as the 'germinal epithelium' as it was earlier mistakenly believed that it could give rise to new germ cells (Auersperg et al. 2001, Szotek et al. 2008). Bipotent stem cells derived from the tunica albuginea of the ovary have been identified with a capacity to give rise to OSE cells, granulosa cells, and germ cells (Bukovsky et al. 2004). Recently, differentiated granulosa cells have been demonstrated to revert to a state of proliferation and long-term survival in the presence of leukemia inhibitory factor (LIF) in culture (Kossowska-Tomaszczuk et al. 2009). Their progressive loss of differentiated characteristics and acquisition of mesenchymal markers besides being indicative of epithelial mesenchymal 
transition (EMT) also suggests a de-differentiation process resulting in reversal to a stem cell state that is experimentally supported by POU5F1 (Oct4) expression and by successful induction of differentiation along the distinct neuronal, chondrocytic and osteoblastic lineages. As with other regenerative tissues, ovarian cancer being derived from such indigenous stem cell populations is thus suggested. The following compilation presents a detailed account of the progress in understanding ovarian cancer as a stem cell disease.

\section{Ovarian cancer and stem cells}

Ovarian cancer is a highly lethal disease estimated to be the most fatal of all gynecologic malignancies. Stem cell dysfunction in adult mammalian ovaries is believed to contribute to diseased states such as ovarian cancer and polycystic ovary syndrome (Tilly \& Rueda 2008). Ovarian cancer appears to represent a group of distinct diseases rather than being a single entity. Three broad histological types based on their cell of origin, with distinct frequencies of occurrence, are identified (Boring et al. 1993, Kusumbe \& Bapat 2008), which are as follows:

Stromal tumors account for only $5 \%$ of overall ovarian tumors and originate from the stromal cells located within the ovarian cortex;

Germ cell tumors originate in the germ cells (GCs) and account for around $5 \%$ of ovarian tumors. The level of malignancy correlates well with extent of differentiation in these tumors; and

Epithelial ovarian carcinoma (EOC) is the most frequent form of the disease, accounts for about $90 \%$ of ovarian cancer cases, and is associated with a very high mortality that is a combined effect of an insidious onset of disease with early nonspecific symptoms combined with a lack of reliable screening tests (Bast et al. 2009). This effectively results in a majority of patients presenting advanced disease at the time of diagnosis. A cause-effect relationship between increased ovulation (early onset of menses, late menopause, and infertility) and cancer incidence has been postulated; consequently, decreased ovulatory cycles (multiple pregnancies and use of oral contraceptives) correlate with lowered risk (Wikborn et al. 1993). Major advances in our understanding and treatment of ovarian cancer have occurred over the past decade leading to identification of optimal combinations of surgery and chemotherapy, yet the long-term cure rate of women with this disease has improved only modestly. Although patients respond initially to standard combinations of surgery and chemotherapy, a large majority succumb later through development of recurrent, therapy-resistant disease (Clarke-Pearson 2009).

Subtypes of each of the above main histological types have further been identified based on the extent of cellular differentiation and molecular features within the tumors as well as on the natural history. More recently, specific subtypes of EOC have been correlated with distinct sites of origin (Kobel et al. 2008, Lawrenson \& Gayther 2009). Current research focuses on appropriating distinct 'molecular signatures' with each type of EOC toward the identification of prognostic and predictive biomarkers that could improve the quality of life for patients and provide better therapeutic options (Tothill 2009). Taken together, ovarian cancer provides a challenge for patients, clinicians, and researchers. One such obstacle has been the isolation and identification of stem cells in tumors that possibly contribute in a major way to therapeutic recalcitrance.

\section{Putative isolation and identification of ovarian CSCs}

The isolation and identification of leukemia-initiating and tumor-initiating cells in other tumors such as breast and brain using multiparametric flow sorting and immune compromised mouse models were critical in demonstrating the contribution of these rare populations of cells to the disease (Lapidot et al. 1994, Al-Hajj et al. 2003, Singh et al. 2004). These strategies were essentially based on knowledge of surface markers, expression patterns, and immunophenotyping of normal stem cells in the organ/tissue, which successfully laid down guidelines for the isolation and identification of CSCs. Analogous to normal stem cells, CSCs are recognized to constitute a small fraction of the total tumor cell mass that is capable of self-renewal and multipotent differentiation, but gives rise to a reproducible tumor phenotype. Lack of precise information regarding similar parameters in normal stem cells in ovarian biology has been a limiting factor in the identification of CSCs in ovarian cancer, which, as discussed earlier, represents a compendium of diseases that are distinct with respect to their histological and molecular characteristics besides varying with respect to their cell of origin. However, using a combination of general stem cell surface markers, capability to generate nonadherent, self-renewing spheroids in vitro, and tumor propagation over several generations in mice have irrevocably established the association of CSCs and epithelial ovarian cancer. Serial passages over 3-4 generations in nude mice have so far been considered as being indicative of self-renewal capabilities of xenografted CSCs (Box 1).

\section{Establishment of an in vitro CSC model derived from ovarian epithelial serous adenocarcinoma}

The first study on the isolation and identification of stemlike cells in ovarian cancer was reported from our lab (Bapat et al. 2005). Since serous adenocarcinoma is the most aggressive and lethal of ovarian cancers, we reasoned that the associated rapid disease progression with respect to metastases and drug resistance could 


\section{Box 1 Ovarian cancer stem cells}

- Stem cells exist in ovarian tumors.

- They can be isolated through approaches based on long-term clonogenicity or expression of specific surface markers or dye exclusion.

- A proof of principle is provided through association of undifferentiated stem cell markers such as POU5F1 (OCT4), NANOG, BMI1, nestin (NES), and ABCG2, followed by differentiation to express ovarian-specific markers and functional assays.

- They conform to CSC assays of functionality in vitro and in vivo, including anchorage-independent growth, self-renewal, and histological specificity of tumors.

- Such CSCs demonstrate unique features that support tumor survival:

- recruitment of endothelial stem cells toward establishment of vasculature in developing tumors;

- epithelial-mesenchymal transition that enhances survival in a tripartite manner - migration away from the primary site, resistance to p53-mediated apoptosis, and enrichment of CSCs in metastases.

involve stem cells that possess these characteristics and thereby, support migration and survival. Toward the identification of such cells, we outlined a classical cell culture approach for the isolation of long-term surviving populations within tumors, and we further analyzed promising single cell-derived clones for expression of stem cell characteristics. Briefly, we developed an in vitro model system comprising of 19 spontaneously immortalized clones derived from an advanced grade patient. Characterization led to the identification that only two of these clones were CSCs, while the remaining 17 were either untransformed or pre-tumorigenic stem cells. All the 19 clones showed the presence of cell surface markers such as c-kit, stem cell factor, CD44, EGFR, and E-cadherin. The two CSC clones were anchorage independent and had an ability to form spheroids in suspension culture and multicellular colonies in soft agar that correlated with in vivo tumorigenicity. Tumors derived from these cells in nude mice were identified to be histopathologically similar to the original human tumor. Such xenografted tumors could be serially passaged through at least three generations in vivo that indicated a capacity for in vivo self-renewal. Our study thus provided evidence of the association of cancer cells harboring a stem cell phenotype, self-renewal, and differentiation abilities with serous ovarian cancer. The data thereby generated provided substantial evidence for the involvement of CSCs in ovarian cancer. While we did include an extensive screening for cell surface markers, we laid more emphasis to functional assays in vitro as well as in vivo. Furthermore, through mitochondrial genome profiling, we identified that the CSCs are probably derived from a single mutant stem cell clone (Wani et al. 2006).

\section{Application of surface markers for the isolation and enrichment of ovarian CSCs}

A cell surface phenotype expressed by normal stem cells in the organ provides a robust and convenient guideline for winnowing the rare CSCs from tumors, since it is based on the premise that CSCs are either derived from normal stem cells or de-differentiated toward this state, and hence would express a similar immunophenotype. This has been the approach applied by several researchers in the field and led to the isolation of leukemic stem cells as CD34+/CD38 - (Lapidot et al. 1994), neural tumor stem cells as CD133 + (Singh et al. 2004), etc. However, altered membrane protein expression patterns are indeed a reality with tumor cells, making it imperative to develop more directed approaches toward identification of specific surface immunophenotypes. In the case of ovarian biology, while relevant information of normal stem cell phenotypes is limiting, over the last few years, researchers have relied on screening several general stem cell markers known to be associated with epithelial stem cells in EOC toward the derivation of a putative phenotype that could possibly be used to isolate and identify ovarian CSCs.

\section{CD133+}

The transmembrane glycoprotein CD133 (prominin-1, PROM1) is expressed by hematopoietic stem cells, epithelial stem cells, and CSCs (Miraglia et al. 1997, Florek et al. 2005) including those in the brain (Singh et al. 2004, Liu et al. 2006), prostate (Collins et al. 2005, Miki et al. 2007), pancreas (Olempska et al. 2007), liver (Yin et al. 2007), skin (Monzani et al. 2007), colon (O'Brien et al. 2007, Ricci-Vitiani et al. 2007) besides 
others. Based on these extensive associations of CD133 with various epithelial tumors, it was hypothesized that they may also be associated with ovarian cancer. Indeed, to date, this appears to be the most studied marker in ovarian cancer.

The investigations began with exploring the expressions of CD133-1 and CD133-2 in normal ovaries, benign ovarian tumors, and ovarian carcinomas. This revealed the presence of cells expressing the two epitopes especially in primary tumors than either in normal, benign or in metastatic tissues (Ferrandina et al. 2008). CD133 + ovarian tumor cells also had high proliferative and clonogenic capabilities than CD133cells. However, this study did not identify any relationship between CD133 expression and clinicopathologic features of the disease, nor was an attempt made to characterize CD133-expressing cells with respect to the defining functional criteria of CSCs.

Another study thereafter supported the putative tumorigenicity of CD133-expressing cells in ovarian tumors and further identified that epigenetic dysregulation of CD133 (PROM1) may be associated with transformation (Baba et al. 2009). This research identified that CD133+ cells derived from ovarian tumors were capable of selfrenewal, and they were associated with increased tumor aggression in xenografts. However, the proposition of these cells being CSCs in ovarian tumors based on the fact that they express a gene expression signature similar to that of other cancer-initiating cells seemed to be an indirect and hence weak support of the concept. Another limitation in accepting CD133 + cells to be ovarian CSCs based on this report was from the findings of the authors that cells devoid of CD133 expression (i.e. CD133 - cells) could also generate tumors in mouse models. However, more recently, another study has identified that tumorderived sorted CD133+ cell populations have an increased tumorigenic capacity over CD133 - cells, and they are capable of regenerating a heterogeneous tumor that is similar to the original patient-derived tumor (Curley et al. 2009). Validation using the in vivo serial transplantation model indicated a capacity of self-renewal. The tumors thus generated were heterogeneous that maintained the histological integrity of the parental tumor. In conclusion, although CD133 initially may have appeared to be a weak candidate, it is increasingly gathering support as an ovarian CSC defining candidate marker.

We had also been intrigued by the presence of CD133 + cells in primary ovarian tumors as well as in metastases, especially since our studies indicated that isolated CD133 + cells lack tumorigenic capabilities. On characterizing these cells in our in vitro model as well as in in vivo model, we identified that CD133+ cells contribute to establishment of tumor vasculature that is critical for tumor cell survival during disease progression (Kusumbe et al. 2009). Using mouse models, we also demonstrated that these cells are actively recruited by functional CSCs for generating tumor microvessels through neovasculogenesis. Our study thus proposed a direct involvement of CD133 expression in disease progression without actually being associated with tumor-initiating capabilities.

\section{$C D 44+/ C D 117+$}

The hyaluronic acid receptor CD44 antigen is expressed in a large number of tumors and is also a marker for CSC subsets in breast tumors, while CD117/KIT (c-kit) is frequently expressed by several stem cells. One of the earliest reports in ovarian CSC biology demonstrated the association of CD44+/CD117+ cells derived from ovarian tumors with CSC characteristics (Zhang et al. 2008). An initial enrichment of stem cells from tumor samples was achieved through exploitation of their capability of anchorage-independent growth to give rise to multilayered spheroids in culture. Following this, developing spheroids were screened for expression of CD44 and CD117 that confirmed their enrichment. Further CD44+/CD177+ cells were found to conform to all the defining characteristics of CSCs outlined earlier. Further support for the candidature of CD44 and CD117 as CSC markers is that they have been identified in normal OSE cells (Parrott et al. 2000), although this association has not been correlated with stem cell characteristics in normal tissues (Cannistra et al. 1995). However, since the cells isolated through this approach conform to stem cell functionalities, the CD44+/CD117+ immunophenotype holds promise as a defining characteristic of ovarian CSCs.

\section{CD44+/MYD88+}

The identification and characterization of $\mathrm{CD} 44+$, MYD88 + cells showing constitutive nuclear factor $\kappa$ B (NFKB) activity, cytokine production, high capacity for repair, chemoresistance to conventional chemotherapies, resistance to tumour necrosis factor $\boldsymbol{\alpha}$-mediated apoptosis, capacity to form spheroids in suspension, and importantly, the ability to recapitulate the original tumor in vivo in relation with mechanisms of chemoresistance in ovarian cancer have been recently reported (Alvero et al. 2009a). This further associates ovarian CSCs with a unique molecular profile that confers distinctive characteristics of CSCs in terms of tumorigenicity, resistance to chemotherapy, differentiation potential in vitro as well as in vivo, and the capacity to promote a proinflammatory microenvironment. The association with MYD88 expression is significant because the normal wound healing/tissue repair processes that have an epidemiological correlation with ovarian cancer (Auersperg et al. 2001) are demonstrated to depend on TLR4-MYD88 signaling; the presence of necrotic centers in tumors has also been associated with a proinflammatory environment. Ovarian CSCs expressing TLR4 and MYD88 would thereby respond to TLR4 ligands by activating NFKB, suggesting that the TLR4 pathway may play a critical role 
in the process of aberrant repair/differentiation triggered by the CSCs.

The same research group in yet another recent report have identified bipotent CD44+/E-cadherin-/CD34cells in ovarian cancer (termed Type I EOC cells) that besides being capable of tumor regeneration also contribute to tumor vascularization by a mechanism that involves inhibitor of $\kappa \mathrm{B}$ kinase (IKK; Alvero et al. $2009 b$ ). These cells secrete pro-angiogenic factors IL6, IL8, and VEGF to generate a conducive microenvironment for neoangiogenesis, besides participating in the process themselves. Identification of such bipotent cells in tumors provides a new dimension to ovarian cancer.

\section{Side population cells as OV-CSCs}

A small subset of cells with the property of active expulsion of certain molecules taken up by cells, e.g. the dye Hoechst 33342, through ATP-binding cassette transporters such as ABCG2/BCRP1, have been identified in several mammalian tissues such as bone marrow, mammary gland, lung, skin, and the cancers of these organs (Alvi et al. 2003, Hirschmann-Jax et al. 2004, Lin \& Goodell 2006, Ambler \& Maatta 2009). This fraction of cells termed 'side population' (SP) can be isolated in flow cytometry-based sorting using their dye efflux properties and further characterized to be associated with stem cell characteristics (Challen \& Little 2006). Stem-like cells have been enriched in the SP cell population from mouse ovaries and characterized (Szotek et al. 2008). The same group has also identified SP populations in ovarian cancer cell lines with a capacity for self-renewal and production of heterologous descendent non-SP populations (Szotek et al. 2006). Such SP cells that demonstrated a significant G1 cell cycle phase arrest in culture, however, could regenerate palpable tumors with lower latency and at a higher frequency than non-SP cells in in vivo models. SP cells were also isolated from human ovarian cancer cell lines and primary human ascites cells from ovarian cancer patients. The finding that SP-derived CSCs were highly resistant to doxorubicin compared with the non-SP cells through ABCG2 expression accounts for evasion mechanisms under chemotherapy, and it further supports the candidature of these cells as being CSCs.

Another detailed study reported recently addressed the optimization of parameters required to identify and isolate the SP populations in ovarian cancer using the human ovarian cancer cell line OVCAR-3 (Gao et al. 2009). Under optimal processing and staining parameters, only $0.9 \%$ of the whole population was sorted as SP cells. This cell fraction alone had a capacity to form holoclones and to express the self-renewal marker POU5F1, and it showed significantly higher colony formation efficiency than the non-SP cells. This report emphasized a need of SP isolation optimization in cell studies whenever these populations are to be considered.

\section{Label retaining, quiescent cells as $\mathrm{OV}$-CSCs}

A defining property of stem cells is that of quiescence; reversible quiescence is critical to normal tissue proliferation kinetics and homeostasis (Yahata et al. 2008). In an analogous manner, CSCs are speculated to remain quiescent, and tumor dormancy is conjectured to correlate with this quiescent cell fraction that is likely to persist post therapy to drive disease relapse (Reya et al. 2001). Despite this realization, quiescence has never actually been demonstrated in CSCs that are classically isolated from tumors exploiting cell surface markers known to be associated with normal stem cells. Classically, label-retaining cell assays that exploit the slow cycling nature of stem cells are widely used for the identification of stem cells (Yue et al. 2005).

Recently, using a convenient readout of retention of fluorescent membrane-labeling dyes such as PKH67/PKH26, we have shown that stem cell activity is enriched in the quiescent fraction of ovarian tumors (Kusumbe \& Bapat 2009). These vital dyes consist of a fluorophore attached to an aliphatic carbon backbone that irreversibly binds to the lipid bilayer on cell membranes. Subsequent to each cell division, the label gets equally partitioned among daughter cells, resulting in the reduction of fluorescence intensity. PKH labeling enables the monitoring of live cells and is useful when subsequent functional analysis of labeled cells is required. Consequently, we demonstrated the reversibility of quiescence in PKH-labeled cells in our study through the functionalities of clonogenicity in vitro and tumorigenicity in vivo. This effectively demarcates quiescent cells from those in other nonproliferating states such as differentiation or senescence. We believe that PKH label retention/quenching to be a highly effective readout for the identification of quiescent cells in all experimental tumors.

\section{Enrichment of ovarian CSCs in situ and tumor survival}

Apart from addressing a mechanistic understanding of tumor formation and propagation, the implications of presence of CSCs in primary tumors as well as in metastasizing cell populations were immediately realized in the context of cancer therapy. A large majority of human ovarian cancers progressively acquire resistance to radiotherapy and chemotherapy, and recur despite complete resection of the tumor. The CSC theory maintains that even if a small number of CSCs remain in situ after therapy, disease recurrence can occur (Reya et al. 2001). On the other hand, if CSCs are eliminated, the possibility of recurrent disease is minimal. Based on an understanding of their characteristics, the refractory response of CSCs to drug and radiation treatments may be attributed to: 
i) resistance to apoptosis,

ii) expression of drug transporters that actively extrude toxic compounds out of the cell,

iii) tumor dormancy and CSC quiescence, and

iv) enrichment of CSCs during disease progression.

Resistance to apoptosis occurs through either inactivation of the intrinsic (several cellular events primarily processed at mitochondria, mutations in tumor suppressor/apoptosis-associated genes, expression of drug resistance/metabolizing genes) or extrinsic pathways (activation of death receptors on the cell surface membranes). Several pathways could be involved in these mechanisms including activation of anti-apoptotic factors, inactivation of pro-apoptotic effectors, and/or reinforcement of survival signals (Gimenez-Bonafe et al. 2009). This aspect has been extensively studied in cancer biology, and several drugs are developed that targetspecific molecules and/or pathways that enhance this response in tumor cells.

Some of the most frequently studied drug transporters associated with acquisition of resistance in normal stem cells as well as in CSCs are multifunctional efflux transporters from the ATP-binding cassette $(A B C)$ gene family (Dean 2009). These contribute to tumor resistance by actively transporting drugs across cell membranes through ATP hydrolysis (Szakács et al. 2006). Three members of the $A B C$ family viz., $A B C G 2 / B C R P, A B C B 1 /$ $\mathrm{P}$-gp/MDR1, and $A B C C$ are reported to be upregulated in several cancers (Donnenberg et al. 2009, Guo et al. 2009, Martin et al. 2009). ABCG2 also mediates efflux of Hoechst dye, and in several instances, the expression of ABCG2 has been correlated with the SP stem cells (Goodell et al. 1996) in normal tissues as well as in tumors.

Quiescence may be a defining property of CSCs with an important association with minimal residual disease and tumor dormancy. As described in a previous section, we have shown that label retention activities enrich quiescent CSCs in experimental ovarian cancer (Kusumbe \& Bapat 2009). This approach outlines an effective experimental tool that can be applied for further studies on understanding the molecular nature of reversible quiescence. Such future endeavors are required to understand tumor dormancy that is linked to CSCs and remains a major hurdle in achieving complete remission.

Enrichment of CSCs in tumors is reported in response to therapy through mechanisms such as EMT, enhanced DNA damage repair, changes in the cellular phenotype between epithelial and mesenchymal states of a cell, viz., EMT and mesenchymal epithelial transition (MET), have key roles in embryonic development, and their importance in the pathogenesis of cancer has also been realized (Peinado et al. 2004). Several transcription factors including SNAI1 (Snail), SNAI2 (SluG), ZEB2 (Sip1) and E47, and TWIST and ZEB1 are associated with repression of the adherens junction molecule E-cadherin that triggers off EMT through dissolution of cellular contacts (Peinado et al. 2007). Emerging data suggest extensive crosstalk among these transcription factors, allowing them to form a signaling network that establishes mesenchymal and stem cell phenotypes (Zavadil et al. 2001, Polyak \& Weinberg 2009). In this context, we have recently reported a detailed study of genome-wide identification of SNAI1 and SNAI2 targets that resolves the specific mechanism underlying the enrichment of stem-like cells post radiation treatment or chemotherapy through EMT (Kurrey et al. 2009). This study identifies an elegant modulation by SNAI1 and SNAI2 toward repressing a newly acquired subset of gene targets under conditions of stress that results in inactivation of p53-mediated apoptosis. Yet another target subset continues to mediate EMT, while de-repression of a smaller group of genes results in acquisition of stem cell-like characteristics. This tripartite functioning in which EMT mediates the escape mechanism to newer, less adverse niches complemented with resistance to apoptosis and acquisition of 'stemness' ensures cell survival under conditions of stress and/or ensures tumor generation that correlates with disease progression. This suggests that such de novo CSC generation arises from a directed de-differentiation of tumor cells that culminates in selective accumulation of quiescent or resistant cells under conditions of stress. Other mechanisms leading to stem cell enrichment under conditions of stress include heightened DNA damage response and repair that contribute significantly to tumor survival (Frosina 2009, Lee et al. 2009).

\section{Future perspectives}

The realization of epithelial ovarian cancer as a stem cell disease has the potential to dramatically change approaches in its treatment. While until a few years ago the identification of these rare cells remained elusive, recent studies have explored various approaches and combinations of cell surface markers to effectively derive these cells from cell models and tumor samples (Fig. 1). This represents a first step toward the development of newer treatment strategies that may be effective in controlling the disease that is considered to be highly refractive to current treatment modalities. Thus, new generation cancer therapies are being developed to specifically target CSCs. Drug targets include surface molecules such as the drug transporters; specific oncoproteins; normal and neoplastic stem cell pathways including WNT, SHH, and $\mathrm{NOTCH}$; survival factors; oxidative stress and related signal transduction pathways; and niche factors (Tu et al. 2009). Several uncertainties, however, remain in understanding the biological and clinical consequences of CSCs. 


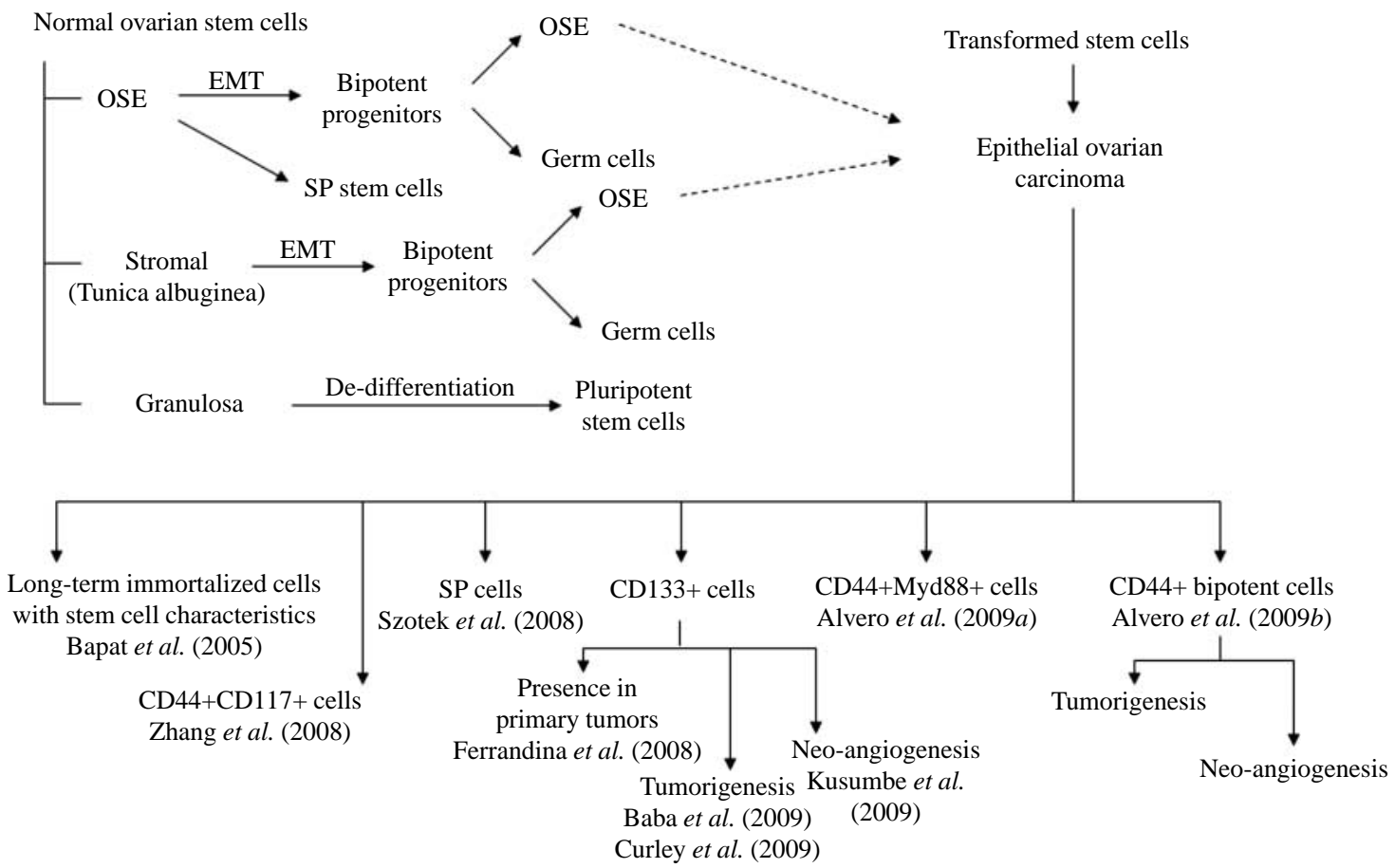

Figure 1 Stem cells identified in the normal ovary and in ovarian tumors.

\section{Which are the real precursors of EOC?}

The mechanistic pathogenesis of human epithelial ovarian tumors is largely unknown due to the diversity in histological subtypes including serous, endometrioid, mucinous, and clear cell cancers. OSE is suggested to be the cell of origin of most ovarian epithelial cancers. Based on the fact that several histological subclasses of EOC exist, this view suggests either a pliability of differentiation of the OSE. Contrarily, evidence of high-grade serous ovarian carcinomas arising as a consequence of deposition on the ovarian surface of transformed cells from the distal part of the fallopian tubes has been recently provided (Crum 2009). Since then the contradicting suggestions have been accounted for through a development perspective considering the coelomic epithelium-derived peritoneum, OSE, and tubal epithelium as a unit derived from the coelomic epithelium during development and exhibiting an enhanced susceptibility to neoplastic changes (Auersperg et al. 2008). Generation of different histotypic tumors from such a common precursor cells might trigger off specific pathways as a function of microenvironmental factors and mutational events. However, it is certain that a better molecular understanding of such precursor cells would improve therapy for patients in the future.

\section{What are the molecular pathways that are disrupted in ovarian carcinogenesis?}

Exploring the relationship and differential behaviors between normal ovarian stem cells and CSCs could resolve mechanisms through which CSCs escape the normal regulatory signals and niche requirements and progress to manipulate other cell types toward forming newer micro- and macro-metastases. The identification of gene regulation mechanisms, co-relations between chromatin modifications and gene expression patterns, and molecules differentially or uniquely expressed by CSCs may provide novel diagnostic and therapeutic markers critical for the elimination of this fraction of cancer cells which is highly resistant to chemotherapy, and therefore, likely responsible for the recurrence and death of the majority of patients diagnosed with ovarian cancer. An example in point is a recent study that reports the stem cell marker ALDH1 as being a favorable prognostic factor in ovarian cancer patients; contrarily, it is associated with poor patient prognosis in breast cancer (Chang et al. 2009). ALDH1 thus may have a different function in ovarian cancer than it does in breast cancer, and identification of its functionality in the normal state may be critical in determining its significance.

\section{Do we need CSC surface markers or CSC biomarkers?}

The success of breast cancer subtyping based on a combination of molecular expression and histology in guiding therapeutic strategies (Sotiriou \& Pusztai 2009) has recently prompted similar investigations in other cancers including ovarian cancer (Brown \& Palmer 2009). While the identification of surface markers has been a major focus in isolating CSCs, it may be pertinent to identify specific CSC-associated genes from 
expression profiles of tumors as biomarkers and evaluate their correlation with patient survival. This could lead to an increased sensitivity and specificity of the prognostic/ predictive value of these biomarkers. To date, several groups have identified gene expression 'signatures' and biomarkers in ovarian cancer (Spentzos et al. 2004, 2005, Mendiola et al. 2008, Murph et al. 2009), and these may further be evaluated in the context of CSC biology.

In conclusion, the current findings initiate an understanding of CSCs in ovarian cancer and further underscore an important need for further evaluation of their origins and mechanisms of survival. Continuing efforts along divergent lines to elucidate the existence, association, and contribution of these cells are necessary to ultimately make the promise of specific targeting of these cells in cancer therapy a reality.

\section{Declaration of interest}

The author declares that there is no conflict of interest that could be perceived as prejudicing the impartiality of this work.

\section{Funding}

This work/review did not receive any specific grant from any funding agency in the public, commercial, or not-for-profit sector.

\section{References}

Al-Hajj M, Wicha MS, Benito-Hernandez A, Morrison SJ \& Clarke MF 2003 Prospective identification of tumorigenic breast cancer cells. PNAS $\mathbf{1 0 0}$ 3983-3988.

Alvero AB, Chen R, Fu HH, Montagna M, Schwartz PE, Rutherford T, Silasi DA, Steffensen KD, Waldstrom M, Visintin I et al. 2009 a Molecular phenotyping of human ovarian cancer stem cells unravels the mechanisms for repair and chemoresistance. Cell Cycle 8 158-166.

Alvero AB, Fu HH, Holmberg J, Visintin I, Mor L, Marquina CC, Oidtman J, Silasi DA \& Mor G $2009 b$ Stem-like ovarian cancer cells can serve as tumor vascular progenitors. Stem Cells 27 2405-2413.

Alvi AJ, Clayton H, Joshi C, Enver T, Ashworth A, Vivanco MM, Dale TC \& Smalley MJ 2003 Functional and molecular characterisation of mammary side population cells. Breast Cancer Research 5 R1-R8.

Ambler CA \& Maatta A 2009 Epidermal stem cells: location, potential and contribution to cancer. Journal of Pathology 217 206-216.

Auersperg N, Wong AS, Choi KC, Kang SK \& Leung PC 2001 Ovarian surface epithelium: biology, endocrinology, and pathology. Endocrine Reviews 22 255-288.

Auersperg N, Woo MM \& Gilks CB 2008 The origin of ovarian carcinomas: a developmental view. Gynecologic Oncology $110452-454$.

Baba T, Convery PA, Matsumura N, Whitaker RS, Kondoh E, Perry T, Huang Z, Bentley RC, Mori S, Fujii S et al. 2009 Epigenetic regulation of CD133 and tumorigenicity of CD133 + ovarian cancer cells. Oncogene 28 209-218.

Bapat SA, Mali AM, Koppikar CB \& Kurrey NK 2005 Stem and progenitorlike cells contribute to the aggressive behavior of human epithelial ovarian cancer. Cancer Research 65 3025-3029.

Bast RC Jr, Hennessy B \& Mills GB 2009 The biology of ovarian cancer: new opportunities for translation. Nature Reviews. Cancer 9 415-428.

Boring CC, Squires TS \& Tong T 1993 Cancer statistics, 1993. CA: a Cancer Journal for Clinicians 43 7-26.
Brown PO \& Palmer C 2009 The preclinical natural history of serous ovarian cancer: defining the target for early detection. PLoS Medicine 6 e1000114.

Bukovsky A, Caudle MR, Svetlikova M \& Upadhyaya NB 2004 Origin of germ cells and formation of new primary follicles in adult human ovaries. Reproduction Biology and Endocrinology 220.

Cannistra SA, Abu-Jawdeh G, Niloff J, Strobel T, Swanson L, Andersen J \& Ottensmeier C 1995 CD44 variant expression is a common feature of epithelial ovarian cancer: lack of association with standard prognostic factors. Journal of Clinical Oncology 13 1912-1921.

Challen GA \& Little MH 2006 A side order of stem cells: the SP phenotype. Stem Cells 24 3-12.

Chang B, Liu G, Xue F, Rosen DG, Xiao L, Wang X \& Liu J 2009 ALDH1 expression correlates with favorable prognosis in ovarian cancers. Modern Pathology 22 817-823.

Clarke-Pearson DL 2009 Clinical practice-screening for ovarian cancer. New England Journal of Medicine 361 170-177.

Collins AT, Berry PA, Hyde C, Stower MJ \& Maitland NJ 2005 Prospective identification of tumorigenic prostate cancer stem cells. Cancer Research 65 10946-10951.

Crum CP 2009 Intercepting pelvic cancer in the distal fallopian tube: theories and realities. Molecular Oncology 3 165-170.

Curley MD, Therrien VA, Cummings CL, Sergent PA, Koulouris CR, Friel AM, Roberts DJ, Seiden MV, Scadden DT, Rueda BR et al. 2009 CD133 expression defines a tumor initiating cell population in primary human ovarian cancer. Stem Cells 27 2875-2883.

Dean M 2009 ABC transporters, drug resistance, and cancer stem cells. Journal of Mammary Gland Biology \& Neoplasia 14 3-9.

Donnenberg VS, Meyer EM \& Donnenberg AD 2009 Measurement of multiple drug resistance transporter activity in putative cancer stem/ progenitor cells. Methods in Molecular Biology 568 261-279.

Ferrandina G, Bonanno G, Pierelli L, Perillo A, Procoli A, Mariotti A, Corallo M, Martinelli E, Rutella S, Paglia A et al. 2008 Expression of CD133-1 and CD133-2 in ovarian cancer. International Journal of Gynecological Cancer 18 506-514.

Florek M, Haase M, Marzesco AM, Freund D, Ehninger G, Huttner WB \& Corbeil D 2005 Prominin-1/CD133, a neural and hematopoietic stem cell marker, is expressed in adult human differentiated cells and certain types of kidney cancer. Cell and Tissue Research 319 15-26.

Frosina G 2009 DNA repair in normal and cancer stem cells, with special reference to the central nervous system. Current Medicinal Chemistry 16 854-866.

Gao Q, Geng L, Kvalheim G, Gaudernack G \& Suo Z 2009 Identification of cancer stem-like side population cells in ovarian cancer cell line OVCAR-3. Ultrastructural Pathology 33 175-181.

Gimenez-Bonafe P, Tortosa A \& Perez-Tomas R 2009 Overcoming drug resistance by enhancing apoptosis of tumor cells. Current Cancer Drug Targets 9 320-340.

Goodell MA, Brose K, Paradis G, Conner AS \& Mulligan RC 1996 Isolation and functional properties of murine hematopoietic stem cells that are replicating in vivo. Journal of Experimental Medicine 183 1797-1806.

Guo Y, Kock K, Ritter CA, Chen ZS, Grube M, Jedlitschky G, Illmer T, Ayres M, Beck JF, Siegmund W et al. 2009 Expression of ABCC-type nucleotide exporters in blasts of adult acute myeloid leukemia: relation to long-term survival. Clinical Cancer Research 15 1762-1769.

Hirschmann-Jax C, Foster AE, Wulf GG, Nuchtern JG, Jax TW, Gobel U, Goodell MA \& Brenner MK 2004 A distinct "side population" of cells with high drug efflux capacity in human tumor cells. PNAS 101 14228-14233.

Kobel M, Kalloger SE, Boyd N, McKinney S, Mehl E, Palmer C, Leung S, Bowen NJ, lonescu DN, Rajput A et al. 2008 Ovarian carcinoma subtypes are different diseases: implications for biomarker studies. PLoS Medicine 5 e232.

Kossowska-Tomaszczuk K, De Geyter C, De Geyter M, Martin I, Holzgreve W, Scherberich A \& Zhang H 2009 The multipotency of luteinizing granulosa cells collected from mature ovarian follicles. Stem Cells 27 210-219.

Kurrey NK, Jalgaonkar SP, Joglekar AV, Ghanate AD, Chaskar PD, Doiphode RY \& Bapat SA 2009 Snail and Slug mediate radio- and chemo-resistance by antagonizing p53-mediated apoptosis and acquiring a stem-like phenotype in ovarian cancer cells. Stem Cells 27 2059-2068. 
Kusumbe AP \& Bapat SA 2008 Ovarian stem cell biology and the emergence of ovarian cancer stem cells. In Cancer Stem Cells, pp 95-110. Ed. S Bapat. Hoboken, NJ: John Wiley \& Sons Inc.

Kusumbe AP \& Bapat SA 2009 Cancer stem cells and aneuploid populations within developing tumors are the major determinants of tumor dormancy. Cancer Research 69 9245-9253.

Kusumbe AP, Mali AM \& Bapat SA 2009 CD133-expressing stem cells associated with ovarian metastases establish an endothelial hierarchy and contribute to tumor vasculature. Stem Cells 27 498-508.

Lapidot T, Sirard C, Vormoor J, Murdoch B, Hoang T, Caceres-Cortes J, Minden M, Paterson B, Caligiuri MA \& Dick JE 1994 A cell initiating human acute myeloid leukaemia after transplantation into SCID mice. Nature 367 645-648.

Lawrenson K \& Gayther SA 2009 Ovarian cancer: a clinical challenge that needs some basic answers. PLoS Medicine 6 e25.

Lee AS, Kahatapitiya P, Kramer B, Joya JE, Hook J, Liu R, Schevzov G, Alexander IE, McCowage G, Montarras D et al. 2009 Methylguanine DNA methyltransferase-mediated drug resistance-based selective enrichment and engraftment of transplanted stem cells in skeletal muscle. Stem Cells 27 1098-1108.

Li C, Lee CJ \& Simeone DM 2009 Identification of human pancreatic cancer stem cells. Methods in Molecular Biology 568 161-173.

Lin KK \& Goodell MA 2006 Purification of hematopoietic stem cells using the side population. Methods in Enzymology 420 255-264.

Liu G, Yuan X, Zeng Z, Tunici P, Ng H, Abdulkadir IR, Lu L, Irvin D, Black KL \& Yu JS 2006 Analysis of gene expression and chemoresistance of CD133 + cancer stem cells in glioblastoma. Molecular Cancer 567.

Martin V, Xu J, Pabbisetty SK, Alonso MM, Liu D, Lee OH, Gumin J, Bhat KP, Colman H, Lang FF et al. 2009 Tie2-mediated multidrug resistance in malignant gliomas is associated with upregulation of $A B C$ transporters. Oncogene 28 2358-2363.

Mendiola M, Barriuso J, Redondo A, Marino-Enriquez A, Madero R, Espinosa E, Vara JA, Sanchez-Navarro I, Hernandez-Cortes G, Zamora P et al. 2008 Angiogenesis-related gene expression profile with independent prognostic value in advanced ovarian carcinoma. PLOS ONE 3 e4051.

Miki J, Furusato B, Li H, Gu Y, Takahashi H, Egawa S, Sesterhenn IA, McLeod DG, Srivastava S \& Rhim JS 2007 Identification of putative stem cell markers, CD133 and CXCR4, in hTERT-immortalized primary nonmalignant and malignant tumor-derived human prostate epithelial cell lines and in prostate cancer specimens. Cancer Research 67 3153-3161.

Miraglia S, Godfrey W, Yin AH, Atkins K, Warnke R, Holden JT, Bray RA, Waller EK \& Buck DW 1997 A novel five-transmembrane hematopoietic stem cell antigen: isolation, characterization, and molecular cloning. Blood 90 5013-5021.

Monzani E, Facchetti F, Galmozzi E, Corsini E, Benetti A, Cavazzin C, Gritti A, Piccinini A, Porro D, Santinami M et al. 2007 Melanoma contains CD133 and ABCG2 positive cells with enhanced tumourigenic potential. European Journal of Cancer 43 935-946.

Murph MM, Liu W, Yu S, Lu Y, Hall H, Hennessy BT, Lahad J, Schaner M, Helland A, Kristensen G et al. 2009 Lysophosphatidic acid-induced transcriptional profile represents serous epithelial ovarian carcinoma and worsened prognosis. PLOS ONE 4 e5583.

O'Brien CA, Pollett A, Gallinger S \& Dick JE 2007 A human colon cancer cell capable of initiating tumour growth in immunodeficient mice. Nature 445 106-110.

Olempska M, Eisenach PA, Ammerpohl O, Ungefroren H, Fandrich F \& Kalthoff H 2007 Detection of tumor stem cell markers in pancreatic carcinoma cell lines. Hepatobiliary \& Pancreatic Diseases International 6 92-97.

Parrott JA, Kim G \& Skinner MK 2000 Expression and action of kit ligand/stem cell factor in normal human and bovine ovarian surface epithelium and ovarian cancer. Biology of Reproduction 62 1600-1609.

Peinado H, Portillo F \& Cano A 2004 Transcriptional regulation of cadherins during development and carcinogenesis. International Journal of Developmental Biology 48 365-375.

Peinado H, Olmeda D \& Cano A 2007 Snail, Zeb and bHLH factors in tumour progression: an alliance against the epithelial phenotype? Nature Reviews. Cancer 7 415-428.
Polyak K \& Weinberg RA 2009 Transitions between epithelial and mesenchymal states: acquisition of malignant and stem cell traits. Nature Reviews. Cancer 9 265-273.

Reya T, Morrison SJ, Clarke MF \& Weissman IL 2001 Stem cells, cancer, and cancer stem cells. Nature 414 105-111.

Ricci-Vitiani L, Lombardi DG, Pilozzi E, Biffoni M, Todaro M, Peschle C \& De Maria R 2007 Identification and expansion of human colon-cancerinitiating cells. Nature 445 111-115.

Singh SK, Hawkins C, Clarke ID, Squire JA, Bayani J, Hide T, Henkelman RM, Cusimano MD \& Dirks PB 2004 Identification of human brain tumour initiating cells. Nature 432 396-401.

Sotiriou C \& Pusztai L 2009 Gene-expression signatures in breast cancer. New England Journal of Medicine 360 790-800.

Spentzos D, Levine DA, Ramoni MF, Joseph M, Gu X, Boyd J, Libermann TA \& Cannistra SA 2004 Gene expression signature with independent prognostic significance in epithelial ovarian cancer. Journal of Clinical Oncology 22 4700-4710.

Spentzos D, Levine DA, Kolia S, Otu H, Boyd J, Libermann TA \& Cannistra SA 2005 Unique gene expression profile based on pathologic response in epithelial ovarian cancer. Journal of Clinical Oncology 23 7911-7918.

Szakács G, Paterson JK, Ludwig JA, Booth-Genthe C \& Gottesman MM 2006 Targeting multidrug resistance in cancer. Nature Reviews. Drug Discovery 5 219-234.

Szotek PP, Pieretti-Vanmarcke R, Masiakos PT, Dinulescu DM, Connolly D, Foster R, Dombkowski D, Preffer F, Maclaughlin DT \& Donahoe PK 2006 Ovarian cancer side population defines cells with stem cell-like characteristics and Mullerian inhibiting substance responsiveness. PNAS 103 11154-11159.

Szotek PP, Chang HL, Brennand K, Fujino A, Pieretti-Vanmarcke R, Lo CC, Dombkowski D, Preffer F, Cohen KS, Teixeira J et al. 2008 Normal ovarian surface epithelial label-retaining cells exhibit stem/progenitor cell characteristics. PNAS 105 12469-12473.

Tilly JL \& Rueda BR 2008 Minireview: stem cell contribution to ovarian development, function, and disease. Endocrinology 149 4307-4311.

Tothill IE 2009 Biosensors for cancer markers diagnosis. Seminars in Cell \& Developmental Biology 20 55-62.

Tu LC, Foltz G, Lin E, Hood L \& Tian Q 2009 Targeting stem cells-clinical implications for cancer therapy. Current Stem Cell Research \& Therapy 4 147-153.

Wani AA, Sharma N, Shouche YS \& Bapat SA 2006 Nuclear-mitochondrial genomic profiling reveals a pattern of evolution in epithelial ovarian tumor stem cells. Oncogene 25 6336-6344.

Wikborn C, Pettersson F, Silfversward C \& Moberg PJ 1993 Symptoms and diagnostic difficulties in ovarian epithelial cancer. International Journal of Gynaecology and Obstetrics 42 261-264.

Yahata T, Muguruma Y, Yumino S, Sheng Y, Uno T, Matsuzawa H, Ito M, Kato S, Hotta T \& Ando K 2008 Quiescent human hematopoietic stem cells in the bone marrow niches organize the hierarchical structure of hematopoiesis. Stem Cells 26 3228-3236.

Yin S, Li J, Hu C, Chen X, Yao M, Yan M, Jiang G, Ge C, Xie H, Wan D et al. 2007 CD133 positive hepatocellular carcinoma cells possess high capacity for tumorigenicity. International Journal of Cancer $\mathbf{1 2 0}$ 1444-1450.

Yue Z, Jiang TX, Widelitz RB \& Chuong CM 2005 Mapping stem cell activities in the feather follicle. Nature 438 1026-1029.

Zavadil J, Bitzer M, Liang D, Yang YC, Massimi A, Kneitz S, Piek E \& Bottinger EP 2001 Genetic programs of epithelial cell plasticity directed by transforming growth factor-beta. PNAS 98 6686-6691.

Zhang S, Balch C, Chan MW, Lai HC, Matei D, Schilder JM, Yan PS, Huang TH \& Nephew KP 2008 Identification and characterization of ovarian cancer-initiating cells from primary human tumors. Cancer Research 68 4311-4320.

Received 31 August 2009

First decision 2 November 2009

Revised manuscript received 5 March 2010

Accepted 16 March 2010 